\title{
The ionizing star of the North America and Pelican nebulae ${ }^{\star \star \star \star}$
}

\author{
F. Comerón ${ }^{1}$ and A. Pasquali ${ }^{2}$ \\ 1 European Southern Observatory, Karl-Schwarzschild-Strasse 2, 85748 Garching, Germany \\ e-mail: fcomeron@eso.org \\ 2 Institute of Astronomy, ETH Hoenggerberg, HPF, 8093 Zurich, Switzerland \\ e-mail: pasquali@phys.ethz.ch
}

Received 4 August 2004 / Accepted 9 September 2004

\begin{abstract}
We present the results of a search for the ionizing star of the North America (NGC 7000) and the Pelican (IC 5070) nebulae complex. The application of adequate selection criteria to the 2MASS $J H K_{\mathrm{S}}$ broad-band photometry allows us to narrow the search down to 19 preliminary candidates in a circle of $0^{\circ} 5$ radius containing most of the L935 dark cloud that separates both nebulae. Follow-up near-infrared spectroscopy shows that most of these objects are carbon stars and mid-to-late-type giants, including some AGB stars. Two of the three remaining objects turn out to be later than spectral type B and thus cannot account for the ionization of the nebula, but a third object, 2MASS J205551.25+435224.6, has infrared properties consistent with it being a mid O-type star at the distance of the nebulae complex and reddened by $A_{V} \simeq 9.6$. We confirm its O5V spectral type by means of visible spectroscopy in the blue. This star has the spectral type required by the ionization conditions of the nebulae and photometric properties consistent with the most recent estimates of their distance. Moreover, it lies close to the geometric center of the complex that other studies have proposed as the most likely location for the ionizing star, and is also very close to the position inferred from the morphology of cloud rims detected in radio continuum. Given the fulfillment of all the conditions and the existence of only one star in the whole search area that satisfies them, we thus propose 2MASS J205551.25+435224.6 as the ionizing star of the North America/Pelican complex.
\end{abstract}

Key words. ISM: HII regions - ISM: individual objects: NGC 7000 - ISM: individual objects: IC 5070 ISM: individual objects: W80

\section{Introduction}

The North America (NGC 7000) and Pelican (IC 5070) nebulae are two of the most nearby and well known diffuse, extended HII regions. Together with the dark lane that separates them, L935 (Lynds 1962) they form the W80 complex (Westerhout 1958). The overall extent and low density of the nebulae indicate that they are relatively evolved, and the absence of compact or ultracompact components in them shows that they do not host massive star formation at present. However, recent and ongoing star formation at intermediate and low masses is revealed by the presence of very young Be and T Tauri stars, already noted by Herbig (1958), and of Herbig-Haro objects (Bally \& Reipurth 2003). Although the distance to the complex has been traditionally controversial (values between 200 and $2000 \mathrm{pc}$ can be found in the literature; see Wendker et al. 1983 and, more recently,

^ Based on observations collected at the Centro Astronómico Hispano-Alemán (CAHA) at Calar Alto, operated jointly by the MaxPlanck Institut für Astronomie and the Instituto de Astrofísica de Andalucía (CSIC).

$\star \star$ Figure 1 is only available in electronic form at http://www. edpsciences.org
Bally \& Reipurth 2003, for references), observations of large samples of stars in the direction of the complex (Laugalys \& Straizys 2002, and references therein) conclusively favor a distance of $(600 \pm 50)$ pc. Their results also demonstrate that both the HII region and L935 are parts of a single entity.

Despite the notoriousness and the proximity of the North America and Pelican nebulae, the star or stars responsible for their ionization have remained unknown until now. The first tentative identification can be traced back to Hubble (1922), who proposed Deneb as the excitation source. Realizing the unsuitability of Deneb's A2Ia spectral type as an ionization source, Sharpless \& Osterbrock (1952) proposed instead the O6V((f)) close binary HD 199579 as a good candidate, but its role as the dominant source of ionization was soon called into question. Herbig (1958) considered it likely that the true ionizing star is actually hidden by L935. A ionizing star different from HD 199579 is also suggested by the geometry (Wendker 1968) and the ionization structure of the nebula (Goudis \& Meaburn 1973; Goudis 1976), which also suggests a spectral type of the ionizing star between $\mathrm{O} 4$ and O5. Following a different approach, high resolution radio continuum observations across the North America/Pelican/L935 complex by Matthews \& Goss (1980) revealed a number of 
sharp rims probably caused by the interaction of the radiation from a central source with dense clumps of gas, similar to the visible bright rims that stand out in $\mathrm{H} \alpha$ images. The positions and morphologies of the rims led Matthews \& Goss (1980) to pinpoint with great accuracy the likely location of the ionizing star, near the geometric center of the nebulae. Near-infrared observations of sources in L935 by Neckel et al. (1980) turned up a heavily reddened, promising candidate ionizing star that was however discarded after further observations by Eiroa et al. (1983), who proved it to be an evolved star unrelated to the complex. Also Bally \& Scoville (1980) produced a list of 11 bright infrared sources among which the ionizing star may be found.

In this paper we use 2MASS observations to photometrically identify possible early-type stars over a large area of L935 that includes the geometric center of the complex and its surroundings, where the ionizing star is most likely located. Since broad-band near infrared photometry alone cannot unambiguously discriminate reddened early-type stars from some classes of cool, evolved luminous stars, we have also obtained $H$ and $K$ band spectroscopy that rules out a physical relationship of most observed stars with the nebula complex. Spectroscopy narrows down our list of candidates to just one moderately reddened star whose photometric and spectroscopic characteristics are in agreement with those of a mid O-type star at the reported distance of the complex. Indeed, we present spectroscopy in the visible that confirms an $\mathrm{O} 5 \mathrm{~V}$ spectral type for this star. We thus propose that this star is the true source of the ionization of the North America and Pelican nebulae.

\section{Field and target selection}

We have carried out our search for the ionizing star of the North America/Pelican complex in a circular area of the sky $0^{\circ} 5$ in radius centered on the coordinates $\alpha(2000)=20^{\mathrm{h}} 55^{\mathrm{m}} 17^{\mathrm{s}}$, $\delta(2000)=+43^{\circ} 47^{\prime} 30^{\prime \prime}$, near the geometric center of the complex. The chosen radius allows for a very generous displacement of the ionizing star with respect to this position. Figure 1 shows that the search area encompasses most of the L935 cloud.

The basic material for our identification of candidate ionizing stars is the 2MASS all-sky survey. The depth of the survey in the $K_{\mathrm{S}}$ magnitude is by far sufficient for the detection of all the candidate ionizing stars even if obscured by $A_{V}=35 \mathrm{mag}$ ( $A_{K}=3.9 \mathrm{mag}$ ), which is the highest value of the extinction derived from the maps of Cambrésy et al. (2002). Figure 2 shows the $\left(H-K_{\mathrm{S}}\right), K_{\mathrm{S}}$ diagram of all the 2MASS stars brighter than $K_{\mathrm{S}}=12$ in the search area. As a first selection, we considered only those stars whose color and magnitude are consistent with them being early-type main sequence stars at the distance of the nebula, assuming an upper limit of $1 \mathrm{kpc}$ to the distance of the complex and a spectral type B0V for the latest possible ionizing star to allow ample margin for uncertainty. Taking intrinsic colors and absolute magnitudes from Tokunaga (2000) and Drilling \& Landolt (2000), and adopting the extinction law of Rieke \& Lebofsky (1985), which is in good agreement with

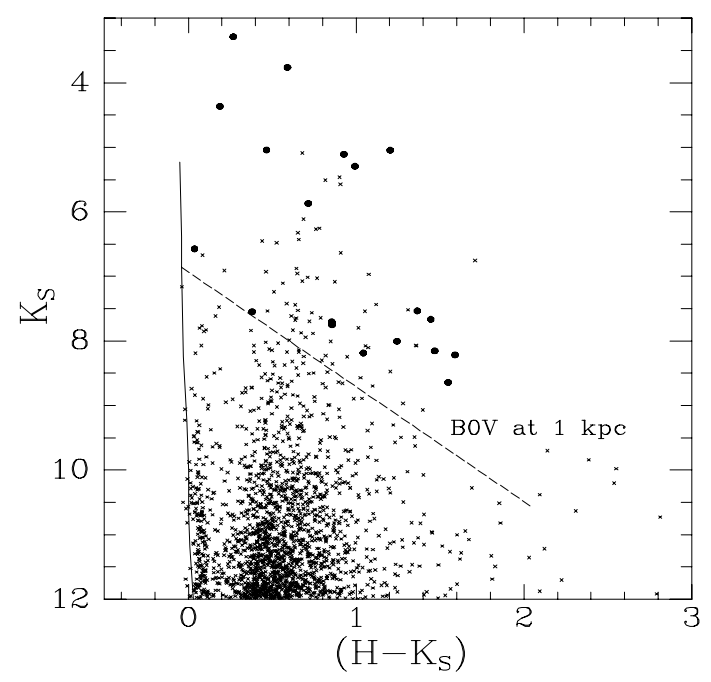

Fig. 2. 2MASS color-magnitude diagram of all the stars brighter than $K_{\mathrm{S}}=12$ found in the search area outlined in Fig. 1 . The solid, nearly vertical line is the unreddened main sequence locus at a distance of $1 \mathrm{kpc}$, with its upper limit corresponding to spectral type O5V. The dashed line is the extinction vector having its origin at the position of an unreddened B0V star at $1 \mathrm{kpc}$, corresponding to the limit given by Eq. (1). We do not consider as possible ionizing stars any objects falling below this line. The filled circles are the stars fulfilling the criterion $Q<0.30$ characterizing (although not exclusively) earlytype stars (see Sect. 2), and for which we obtained $H$ and $K$ band spectroscopy.

the 2MASS color-color diagram of the region (Cambrésy et al. 2002), we selected as candidates stars fulfilling the condition

$K_{\mathrm{S}}<7.0+1.78\left(H-K_{\mathrm{S}}\right)$.

A second selection can be performed taking into account that the intrinsic colors of early-type and most late-type stars cause them to lie along separated sequences in the $\left(H-K_{\mathrm{S}}\right),(J-$ $H$ ) diagram when reddened by an arbitrary amount, as clearly illustrated for instance by the color-color diagram of the nearby Cygnus OB2 association presented by Comerón et al. (2002). Such difference implies that at any given $\left(H-K_{\mathrm{S}}\right)$ red giant stars are redder in $(J-H)$ than early-type stars by about 0.4 mag, a difference greatly exceeding the typical 2MASS photometric uncertainties in the magnitude ranges of relevance to this work. It is thus possible to separate early-type stars from background red giants by using the reddening-free quantity

$Q=(J-H)-1.70\left(H-K_{\mathrm{S}}\right)$.

Early-type stars are expected to be characterized by $Q \simeq 0$, whereas red giants should have $Q \simeq 0.5$. We have thus further restricted our list of possible ionizing stars by selecting only those with $Q<0.30$, which again provides sufficient allowance for individual deviations from the average intrinsic colors at a given spectral class and for photometric uncertainties. Furthermore, we have excluded objects with $H-K_{\mathrm{S}}>2$, since these are faint, very red objects whose 2 MASS $J$-band photometry becomes unreliable. Since $\left(H-K_{\mathrm{S}}\right)>2$ implies $A_{V}>32$ for an early-type star with normal colors (which is very similar to the highest values in the extinction maps of 
Cambrésy et al. 2002), imposing $H-K_{\mathrm{S}}<2$ is not expected to exclude any candidate ionizing stars in the search area. Although $Q<0.30$ should select all the early-type stars in the region (including those displaying near-infrared excess caused by circumstellar material, which would further decrease the value of $Q$ ) the sample is potentially contaminated by oxygenrich AGB stars and carbon-rich giants, as both classes of objects can display $J H K_{\mathrm{S}}$ colors indistinguishable from those of normal, reddened early-type stars (Bessell \& Brett 1988). The combined application of the conditions given by Eq. (1) and by $Q<0.30$ yields a sample of 19 objects spread over the search area, a number permitting easy spectroscopic follow-up. These 19 objects are marked as filled circles in Fig. 2.

\section{Spectroscopic observations}

\subsection{Infrared spectroscopy}

Spectra of the 19 selected objects were obtained on the night of 30/31 July 2004 from the Calar Alto observatory using MAGIC, the near-infrared camera and spectrograph at the $2.2 \mathrm{~m}$ telescope. The spectra were obtained using the resinreplica grism providing a resolution $\lambda / \Delta \lambda=240$ over the 1.50 $2.40 \mu \mathrm{m}$ range with the $1^{\prime \prime}$ slit used. Each star was observed at six positions along the slit, with exposure times per position ranging from $20 \mathrm{~s}$ (stacking 10 integrations of $2 \mathrm{~s}$ ) for the brightest stars to $60 \mathrm{~s}$ (stacking 20 integrations of $3 \mathrm{~s}$ ) for the faintest. The extraction and calibration of the spectra was carried out using dedicated IRAF scripts. The frames obtained at consecutive slit positions were subtracted from each other to cancel out the sky contribution to the spectrum, and were divided by a flat field frame. A one-dimensional object spectrum was then extracted from each sky-subtracted, flat-fielded frame. Wavelength calibration of each individual spectrum was performed using the $\mathrm{OH}$ airglow lines in each frame as a reference (Oliva \& Origlia 1992). The wavelength-calibrated spectra extracted at each sky position were then coadded, with deviant pixels due to detector defects or cosmic ray hits automatically clipped off. Cancellation of telluric features was achieved by ratioing the object spectra by those of the nearby G5IV star HD 190771, which is expected to be featureless at the resolution employed, reduced in the same manner. Finally, relative flux calibration was performed by multiplying the reduced spectra by that of a $5700 \mathrm{~K}$ blackbody, which should be a good approximation to the spectral energy distribution of an unreddened G5IV star in the wavelength range covered by our spectra.

\subsection{Visible spectroscopy}

Spectroscopy in the blue of two selected stars, referred to as \#4 and \#11 in Sect. 4, was performed on the night of 2/3 August 2004 using CAFOS, the visible imager and spectrograph at the $2.2 \mathrm{~m}$ telescope on Calar Alto. A grism was used covering the range shortwards of $\lambda=6350 \AA$ at a resolution of $\lambda / \Delta \lambda=1000$ and with a $1 " 5$ slit. The observations of Star \#4 amounted to $145 \mathrm{~min}$ of exposure time split into five individual exposures. Spectra produced by three lamps of $\mathrm{HgCd}, \mathrm{He}$, and $\mathrm{Rb}$ were

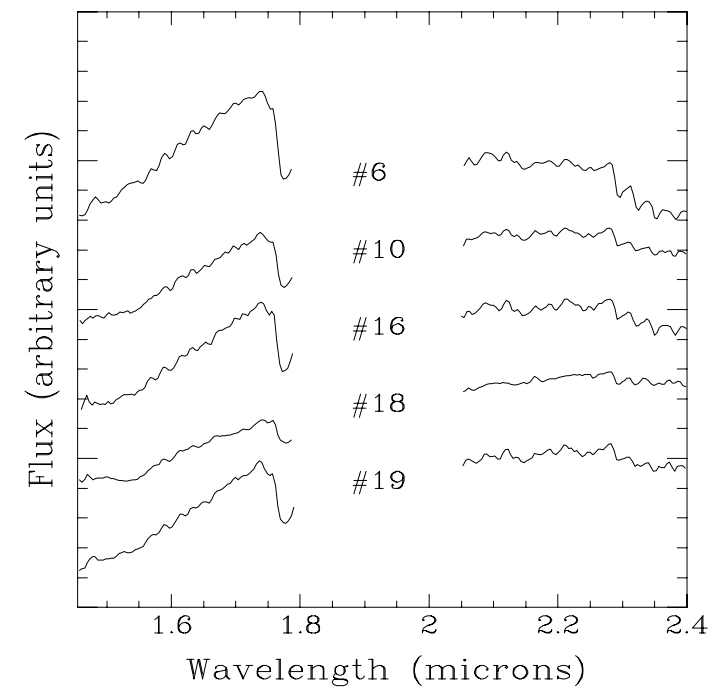

Fig. 3. Infrared spectra of carbon stars included in our sample, characterized by the sharp flux drop at $\lambda=1.77 \mu \mathrm{m}$. The region between $1.85 \mu \mathrm{m}$ and $2.05 \mu \mathrm{m}$ is heavily affected by telluric $\mathrm{H}_{2} \mathrm{O}$ and $\mathrm{CO}_{2}$ absorption and has been removed.

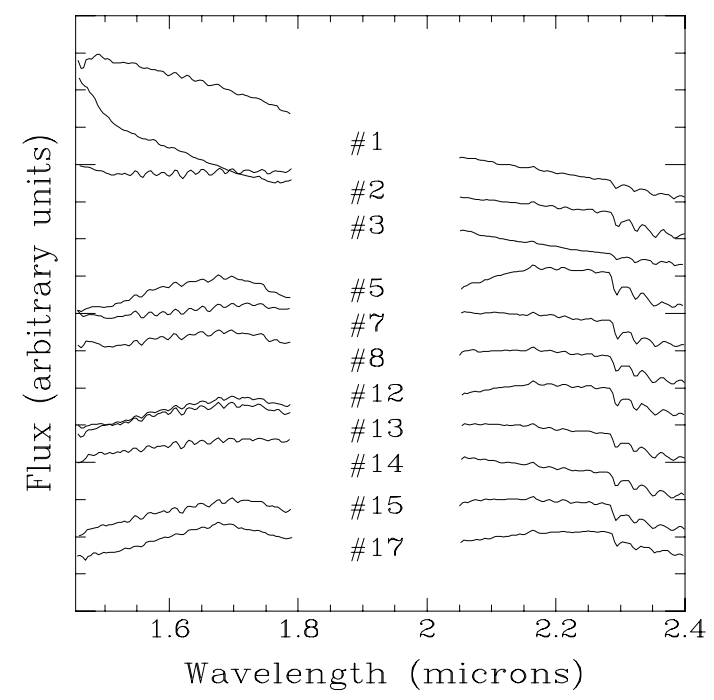

Fig. 4. Infrared spectra of mid- or late-type objects included in our sample other than carbon stars, characterized by the appearance of $\mathrm{CO}$ bandheads longwards of $2.29 \mu \mathrm{m}$. The region between $1.85 \mu \mathrm{m}$ and $2.05 \mu \mathrm{m}$ is heavily affected by telluric $\mathrm{H}_{2} \mathrm{O}$ and $\mathrm{CO}_{2}$ absorption and has been removed.

taken between consecutive exposures for wavelength calibration, in order to minimize the effects of instrument flexure. The spectrum of Star \#11, much brighter in the blue, consisted of one single integration of $30 \mathrm{~min}$. The frames were subtracted from bias and divided by a spectroscopic flat field, and the spectra were subsequently extracted from each one of them. The individual wavelength-calibrated spectra of Star \#4 were coadded after identification and removal of cosmic ray hits. Due to the steep slope of the spectra of these considerably reddened stars, their continuum was normalized to unity in order to facilitate feature recognition and comparison to spectral atlases. 
Table 1. Positions, photometry, and near-infrared spectral types of the observed stars.

\begin{tabular}{lcccccc}
\hline \hline Star & $\alpha(2000)$ & $\delta(2000)$ & $K_{\mathrm{S}}$ & $(J-H)$ & $\left(H-K_{\mathrm{S}}\right)$ & spectrum \\
\hline 1 & $20: 55: 26.5$ & $+43: 22: 02.0$ & 3.286 & 0.721 & 0.268 & Mid- to late-type \\
2 & $20: 57: 41.5$ & $+43: 35: 55.5$ & 3.762 & 1.274 & 0.590 & Red giant \\
3 & $20: 56: 24.4$ & $+43: 54: 02.4$ & 4.366 & 0.485 & 0.188 & Mid- to late-type \\
4 & $20: 55: 51.3$ & $+43: 52: 24.7$ & 5.041 & 0.849 & 0.466 & Early-type \\
5 & $20: 55: 42.3$ & $+43: 52: 14.1$ & 5.045 & 2.094 & 1.203 & AGB \\
6 & $20: 54: 47.6$ & $+43: 22: 41.8$ & 5.106 & 1.749 & 0.927 & Carbon \\
7 & $20: 54: 14.3$ & $+44: 05: 25.7$ & 5.293 & 1.729 & 0.993 & Red giant \\
8 & $20: 55: 41.6$ & $+44: 17: 06.0$ & 5.869 & 1.501 & 0.715 & AGB \\
9 & $20: 55: 16.6$ & $+43: 47: 25.6$ & 6.573 & 0.202 & 0.037 & Early-type \\
10 & $20: 57: 25.7$ & $+43: 57: 35.0$ & 7.534 & 2.296 & 1.364 & Carbon \\
11 & $20: 55: 55.4$ & $+43: 21: 59.3$ & 7.547 & 0.663 & 0.380 & A-type \\
12 & $20: 55: 42.6$ & $+43: 55: 28.1$ & 7.665 & 2.589 & 1.445 & AGB \\
13 & $20: 52: 41.0$ & $+43: 49: 25.0$ & 7.704 & 1.742 & 0.854 & AGB \\
14 & $20: 53: 42.9$ & $+43: 34: 39.9$ & 7.750 & 1.685 & 0.856 & Red giant \\
15 & $20: 54: 44.3$ & $+44: 15: 10.3$ & 8.005 & 2.306 & 1.243 & AGB \\
16 & $20: 55: 21.5$ & $+44: 00: 27.9$ & 8.154 & 2.693 & 1.468 & Carbon \\
17 & $20: 55: 34.3$ & $+43: 26: 37.1$ & 8.187 & 1.782 & 1.043 & AGB \\
18 & $20: 54: 03.6$ & $+44: 02: 54.5$ & 8.216 & 2.486 & 1.589 & Carbon \\
19 & $20: 54: 39.8$ & $+43: 52: 15.6$ & 8.642 & 2.831 & 1.548 & Carbon \\
\hline
\end{tabular}

\section{Results}

Our near-infrared spectroscopic results are summarized in Table 1. The vast majority of the spectra of the 19 stars observed are found to display the distinctive characteristics of cool photospheres: $\mathrm{CO}(2,0)$ absorption bands starting at $2.293 \mu \mathrm{m}$ are visible in the spectra of 16 of the objects. The abundant presence in our sample of carbon stars ( 5 objects easily distinguishable by the prominent $\mathrm{C}_{2}$ feature at $1.77 \mu \mathrm{m}$ ) and AGB stars ( 6 objects, also easy to identify due to the extended wings of the broad water vapor feature separating the $H$ and $K$ bands) is due to their intrinsic colors similar to those of early-type stars as noted in Sect. 2. The misclassification of the first three objects as possible early-type stars in the photometry-selected sample is due to saturation effects in the 2MASS $K$-band photometry; we decided to keep these three objects in the spectroscopic sample since their brightness and, in the case of object \#2, the reddening, were in principle compatible with the photometric properties expected for the ionizing star. The misclassification of object \#7 cannot be explained in those grounds, and it may be due instead to intrisic infrared excess emission. The spectra of the cool objects, split between carbon stars on one side, and AGB stars and other mid-to-late type stars on the other, are shown in Figs. 3 and 4 respectively.

In principle, the most interesting stars from the viewpoint of our search are \#4, \#9, and \#11, which display infrared spectra characteristic of early-type stars. A spectral classification of Star \#9 (=HD 199373) as F5V exists in the literature (Fehrenbach 1961), which agrees with the spectral type derived from its Strømgren photometric indices (Jordi et al. 1996). Its color and spectral type suggest that it is a nearby unreddened star, in agreement with the distance of $70 \mathrm{pc}$ measured by Hipparcos, as well as with the featureless infrared spectrum that we obtain (Fig. 5). This is therefore a foreground star whose relationship to the complex under discussion can

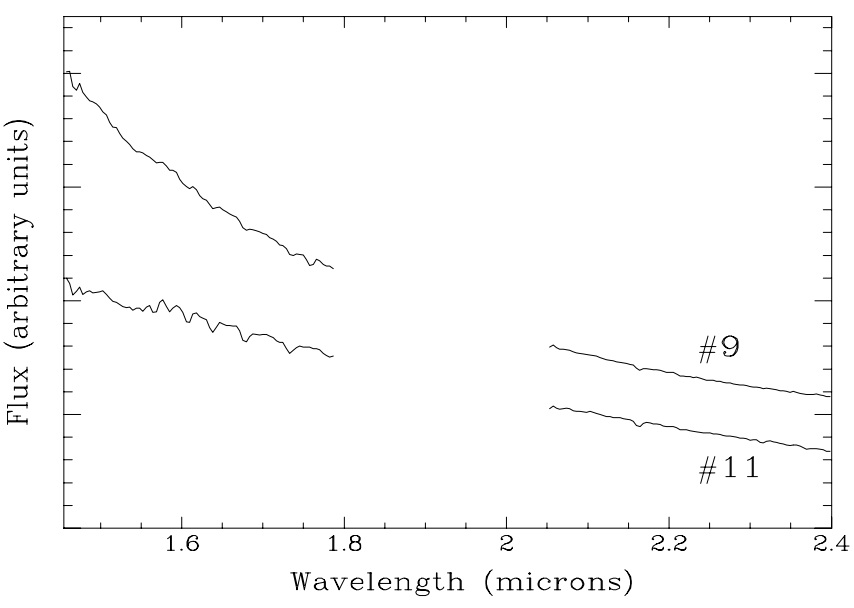

Fig. 5. Two stars with early-type spectra ruled out as possible ionizing sources. Star \#9 is an unobscured star classified in the visible as F5V, while the prominent Br $\gamma$ lines unambigously classify Star \#11 as an early A type. The reddening towards Star \#11 indicates that it lies at the distance of L935 or beyond, suggesting that it is a giant or supergiant. The region between $1.85 \mu \mathrm{m}$ and $2.05 \mu \mathrm{m}$ is heavily affected by telluric $\mathrm{H}_{2} \mathrm{O}$ and $\mathrm{CO}_{2}$ absorption and has been removed.

be safely ruled out. On the other hand, Star \#11, whose spectrum is also shown in Fig. 5, displays prominent $\mathrm{Br} \gamma$ absorption lines whose equivalent depths are near the peak defined by early A types (Meyer et al. 1998), indicating a spectral classification between A0 and A4, which discards it as a possible ionizing star. Its infrared colors are redder than those intrinsic of a A-type star, indicating an extinction of $A_{V} \simeq 6$ mag. The visible spectrum, presented in Fig. 6, confirms the early $A$ type of the star but places it above the main sequence as derived from its relatively narrow Balmer series lines, lacking the broad wings that characterize A stars of luminosity class V (e.g. 


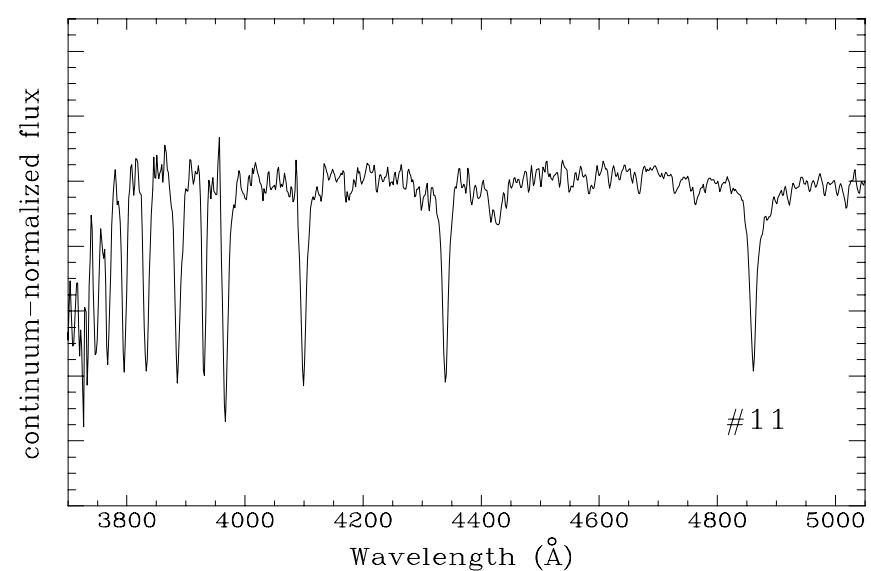

Fig. 6. Spectrum of Star \#11, showing the Balmer lines $\mathrm{H} \beta$ and higher. The strength of the hydrogen lines, and the lack of helium or metallic lines other than the interstellar CaII K line at $\lambda=3933 \AA$, confirm that this is an early-type A star. The Balmer line wings are much less prominent than in luminosity class $\mathrm{V}$ stars, but broader than in the supergiant Deneb (A2Ia), thus suggesting a luminosity class III.

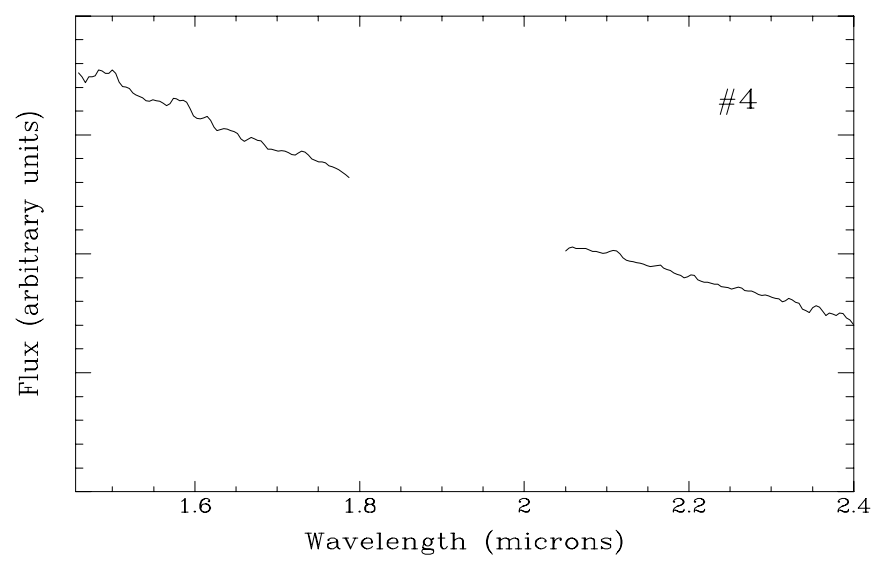

Fig. 7. Infrared spectrum of Star \#4 = J205551.3+435225, which we propose as the ionizing star for the North America and Pelican nebulae. The lack of absorption lines at the resolution of this spectrum is consistent with a $\mathrm{O}$ or early $\mathrm{B}$ type, or with a $\mathrm{F}$ or later type. The former possibility is favored by the $J H K_{\mathrm{S}}$ colors. The region between $1.85 \mu \mathrm{m}$ and $2.05 \mu \mathrm{m}$ is heavily affected by telluric $\mathrm{H}_{2} \mathrm{O}$ and $\mathrm{CO}_{2}$ absorption and has been removed.

Jaschek \& Jaschek 1987). A main-sequence classification is also ruled out by the fact that the absolute magnitude of a A0V star, $M_{K}=0.65$ (Drilling \& Landolt 2000), would imply a distance of only $175 \mathrm{pc}$. Since the only candidate to produce such a high obscuration in the direction of the star is the L935 cloud, a luminosity class $\mathrm{V}$ would require an even closer distance of the cloud from the Sun, which does not seem plausible in view of other evidence, in particular the reddening distance measurements of Laugalys \& Straizys (2002) that set a firm lower limit of $400 \mathrm{pc}$. On the other hand, a comparison to a spectrum of the nearby A supergiant Deneb obtained with the same instrumental setup shows that the Balmer lines of Star \#11 are broader, suggesting a luminosity class III for the latter and consistent with its location behind L935 and the W80 complex.

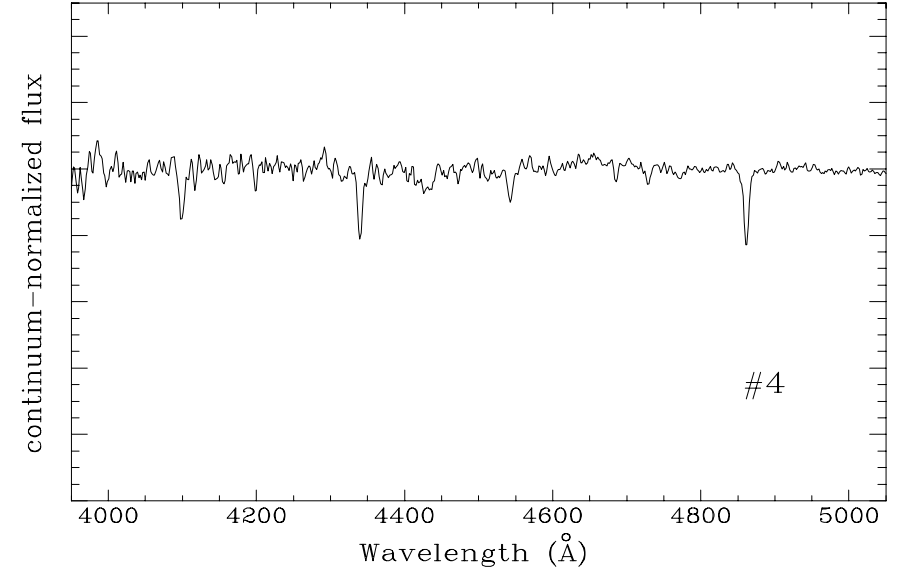

Fig. 8. Visible spectrum of Star \#4 $=\mathrm{J} 205551.3+435225$. Note the appearance of a strong HeII line at $\lambda=4543 \AA$ and the correspondingly weak neighbouring HeI line at $\lambda=4471 \AA$, which indicate an O5 spectral classification. The broad feature centered near $\lambda=4428 \AA$ is due to interstellar absorption.

\subsection{The ionizing star candidate}

The photometric and spectroscopic characteristics of the third early-type star, \#4 (hereafter referred to as J205551.3+435225, after its sexagesimal denomination in the 2MASS catalog, 2MASS J205551.25+435224.6), make it an ideal candidate ionizing star of the North America/Pelican complex. The star is bright in the infrared, and was included by Bally \& Scoville (1980) in the list of sources with near-infrared characteristics consistent with an early spectral type as their source \#10. Assuming an intrinsic infrared color $\left(H-K_{\mathrm{S}}\right)=-0.05$ characteristic of a mid O-type star, the 2MASS color $\left(H-K_{\mathrm{S}}\right)=$ $0.516 \pm 0.034$ implies an extinction $A_{K}=0.92 \pm 0.06$, or $A_{V}=8.2 \pm 0.5$. The extinction can also be estimated from the $B$ magnitude obtained from the USNO catalog, where the star is listed as having $B=15.5, R=11.7$. Adopting $(B-K)=-1.23$ and a normal extinction law in the visible with $R=A_{V} / E[B-V]=3.1$ we obtain $A_{V} \simeq 9.6$, which we take to be more reliable due to the wider baseline in wavelength. The agreement with the value derived from the infrared color is good, taking into account that the estimated extinction is potentially affected by uncertainties stemming from the assumption of a normal extinction law (especially in the visible, due to the increasing dependency on grain properties as one moves to shorter wavelengths) and from possible differences between assumed and actual intrinsic stellar colors. The value is also in good agreement with the extinction derived by Cambrésy et al. (2002) in that direction. The featurelesness of the infrared spectrum (Fig. 7) indicates a spectral type either no later than early B or later than early $\mathrm{F}$, but the $Q=0.06$ derived from the $J H K_{\mathrm{S}}$ photometry strongly favors the early option.

The resolution and signal-to-noise ratio of our infrared spectrum does not allow us to further refine its classification (Hanson et al. 1996; Meyer et al. 1998). However, the moderate reddening makes $\mathrm{J} 205551.3+435225$ accessible to visible spectroscopy in the blue, where far more accurate classification is possible. Besides $\mathrm{H} \beta$ and $\mathrm{H} \gamma$, the spectrum (Fig. 8) displays prominent HeII Pickering and Fowler lines at $\lambda=4200 \AA$, 
$4542 \AA$, and $4686 \AA$, which leave no doubt concerning the classification of J205551.3+435225 as a O-type star. Also easily identifiable are the interstellar features at $\lambda=4428 \AA, 4727 \AA$ (not to be mistaken by HeI at $\lambda=4713 \AA$, which is not visible in our spectrum), and $4762 \AA$ with an intensity similar to that observed in other considerably obscured O-type stars (Hanson 2003). In contrast with the prominence of the HeII lines, there is a nearly total absence of HeI lines. In particular the most intense $\mathrm{HeI}$ in this interval, at $\lambda=4471 \AA$, is barely visible. Its intensity ratio with respect to the nearby HeII line at $4542 \AA$, which is a sensitive spectral subtype indicator in this range (Walborn \& Fitzpatrick 1990), clearly indicates that J205551.3+435225 cannot be later than spectral type O5. On the other hand, the absence of NV lines and the ratio of the two HeII lines at $4686 \AA$ and $4542 \AA$, both of which are luminosity class indicators in the mid-O range (Walborn \& Fitzpatrick 1990), rule out a luminosity class I or III. We thus classify J205551.3+435225 as a O5V star, but note that an even earlier spectral subclass is not excluded.

Adopting the O5V spectral type for J205551.3+435225, and taking average absolute magnitudes and colors for stars of this type from the compilations of Drilling \& Landolt (2000) and Tokunaga (2000), we obtain a distance of $610 \mathrm{pc}$ to this star, in excellent agreement with the most recent determinations of the distance to the North America/Pelican complex. Moreover, the position of the star (shown in Fig. 9) is only 6'2 away from the location proposed by Matthews \& Goss (1980) from their radio continuum maps, and barely outside the $5^{\prime}$ uncertainty radius suggested by them. The ridges, and their preferential orientation facing the star, are well visible at the $1^{\prime}$ resolution of the Canadian Galactic Plane Survey $1420 \mathrm{MHz}$ radio continuum map displayed in the lower panel of Fig. 9.

The O5V spectral type and $610 \mathrm{pc}$ distance also compare favorably with the bulk properties of the complex. Its approximate angular diameter of $3^{\circ}$ corresponds to a physical diameter of $32 \mathrm{pc}$. The electron density $n_{\mathrm{e}}$ can be inferred from the optically thin radio continuum flux and the physical size by relating the emission measure and the brightness temperature, under the assumption of a uniform density and the adoption of a given electron temperature (Wendker 1968). The uniform density distribution seems to be a good approximation given the remarkable lack of small-scale structure in the region, pointed out by Wendker et al. (1983). Correcting Wendker's (1968) calculation for the updated distance of $610 \mathrm{pc}$, we obtain $n_{\mathrm{e}}=$ $11.6 \mathrm{~cm}^{-3}$. A similar value, $n_{\mathrm{e}} \simeq 10 \mathrm{~cm}^{-3}$, is obtained using the expression given by Goudis (1976) for that same distance and correcting for the smaller angular size adopted in that work. Assuming the HII region to be ionization-bounded, as suggested by the molecular shell that surrounds it, the required ionizing flux is $S_{\mathrm{UV}}=0.9 \times 10^{49} \mathrm{~s}^{-1}$, which is approximately one third of the modeled ionizing flux of a O5V star (Schaerer $\&$ de Koter 1997). The agreement can be considered as good given the simplified modeling of the nebula as a homogeneous ionized sphere, although the factor-of-three excess of ionizing photons produced by the star hints at the complex being at least partly density-bounded.

The X-ray source 1RXS J205549.4+435216, faintly detected in the ROSAT All-Sky Survey (Voges et al. 1999), has

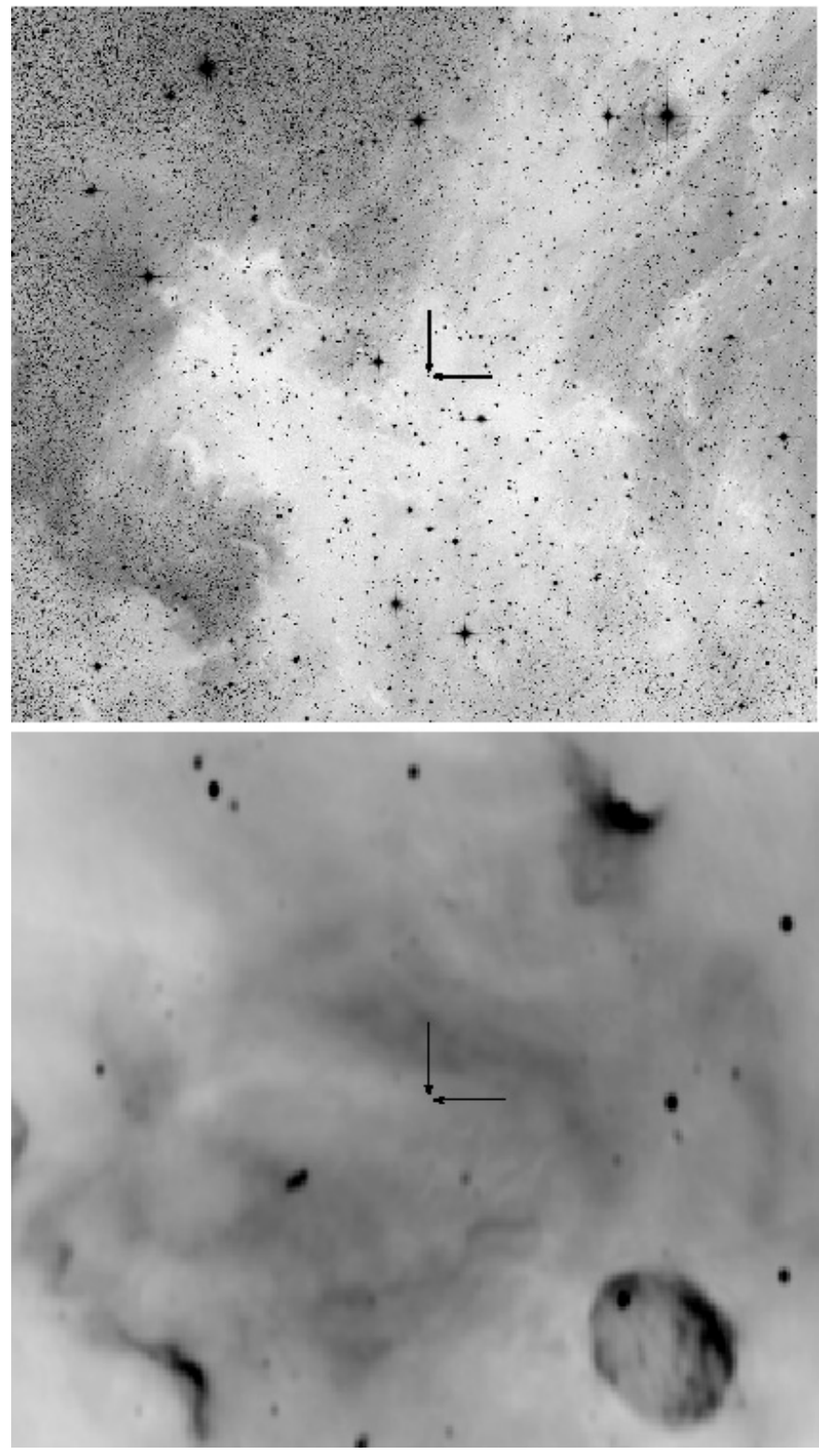

Fig. 9. A detailed visible view of the L935 dark cloud separating the North America and the Pelican nebulae, marking the position of Star $\# 4=\mathrm{J} 205551.3+435225$. The upper panel shows a $94^{\prime} 2 \times 84^{\prime} 9$ section of the same image as Fig. 1 centered on J205551.3+435225. The lower panel displays the same field as observed at the $1420 \mathrm{MHz}$ radio continuum, in a grey scale where white corresponds to a brightness temperature $T_{\mathrm{b}}=5 \mathrm{~K}$ and black to $T_{\mathrm{b}}=25 \mathrm{~K}$. The bubble-like structure on the lower right is the background supernova remnant G84.2-0.8 (Matthews et al. 1977). The radio data are from the Canadian Galactic Plane Survey (Taylor et al. 2003).

a nominal position only $22^{\prime \prime}$ away from our proposed ionizing star. The offset is somewhat larger than the quoted uncertainty of $14^{\prime \prime}$ in the X-ray position, but we still consider it possible that the X-ray source is the counterpart of $\mathrm{J} 205551.3+435225$. Its very high X-ray hardness ratio, $H R 1=+0.92 \pm 0.09$, can be well explained by the large foreground extinction that we derive from the infrared and visible colors, which absorbs the bulk of the soft X-ray emission from the star. Assuming the temperature $T_{X}$ of the X-ray emitting gas to be $k T_{X}=0.6-0.7 \mathrm{keV}$, as is 
typical of mid O-type stars (Berghöfer et al. 1996), we can estimate the conversion factor between ROSAT PSPC count rate and unabsorbed X-ray flux as $\sim 2 \times 10^{-11} \mathrm{erg}_{\text {count }}{ }^{-1} \mathrm{~cm}^{-2}$. We note however that this conversion factor, which we obtain adopting an extinction $A_{V}=3.3 E(B-V)=8.2$ (where $E(B-V)$ is the color excess) and a column density $N_{\mathrm{H}}=5.9 \times 10^{21} \mathrm{~cm}^{-2}$ $E(B-V)$ (Berghöfer et al. 1996), involves an extrapolation of Fig. 3 of Berghöfer et al. (1996) to $\log N_{\mathrm{H}}\left(\mathrm{cm}^{-2}\right) \simeq 22.2$ in the steepest part of the relevant curves, and is thus uncertain perhaps by as much as $50 \%$. The tabulated ROSAT PSPC count rate of $8.2 \times 10^{-2}$ counts s ${ }^{-1}$ implies a X-ray luminosity $7.1 \times 10^{31} \mathrm{erg} \mathrm{cm}^{-2} \mathrm{~s}^{-1}$ at the distance of $600 \mathrm{pc}$. Assuming a typical luminosity for a O5V star $L_{\mathrm{bol}}=7.9 \times 10^{5} L_{\odot}$ (Drilling \& Landolt 2000), this yields $\log \left(L_{\mathrm{X}} / L_{\mathrm{bol}}\right)=-7.6$, which is in fairly good agreement with the average properties of luminous O-type stars (Berghöfer et al. 1996).

We finally note that J205551.3+435225 is remarkably isolated, as implied by the fact that our selection criteria would have picked up any other star earlier than $\mathrm{B} 2 \mathrm{~V}$ existing within the search radius. If the cross-section $\left(85 \mathrm{pc}^{2}\right.$ at the distance of $600 \mathrm{pc}$ ) sampled a volume large enough to contain a typical representation of the initial mass function (IMF), one could estimate the expected number of stars with spectral types between $\mathrm{B} 2 \mathrm{~V}$ and $\mathrm{O} 5 \mathrm{~V}$ from its ratio with respect to the number of stars O5V and earlier expected from the IMF:

$N(\mathrm{~B} 2 \mathrm{~V}>S>05 \mathrm{~V})=N(S \leq \mathrm{O} 5 \mathrm{~V}) \frac{\int_{M(\mathrm{~B} 2 \mathrm{~V})}^{M(\mathrm{~V})} \Phi(M) \mathrm{d} M}{\int_{M(\mathrm{O} 5 \mathrm{~V})}^{\infty} \Phi(M) \mathrm{d} M}$

where $S$ represents the main-sequence spectral type, $\Phi(M)$ is the functional form of the IMF, $M$ is the mass of the star, and $M(S)$ is the mass corresponding to spectral type $S$. The symbols $<$ and $>$ here applied to spectral types are meant to represent "earlier than" and "later than" respectively. Using a Salpeter mass function $\Phi(M) \mathrm{d} M \propto M^{-2.35} \mathrm{~d} M$ and $N(S \leq$ $\mathrm{O} 5 \mathrm{~V})=1$, we would thus expect approximately another 10 early-type stars within our search radius, assuming $M(\mathrm{~B} 2 \mathrm{~V})=$ $10 M_{\odot}$ and $M(\mathrm{O} 5 \mathrm{~V})=60 M_{\odot}$. This in contrast with the lack of such stars that we find in the search area, most probably indicating that the stellar density in the region is too small for our search radius of $5.2 \mathrm{pc}$ to provide a representative sampling of the upper mass function and that J205551.3+435225 can thus be considered as a truly isolated star. We note that, given the modest size of the complex included in our search radius, the isolation of $\mathbf{J} 205551.3+435225$ does not rule out the possible existence of other $\mathrm{OB}$ stars further away in the complex, and in particular of at least some of the O8-B0 stars invoked by Wendker et al. (1983) to account for the detailed structure of the HII region.

\section{Summary}

We have presented a search for the ionizing star of the North America and Pelican nebulae in a large area $\left(0^{\circ} 5\right.$ radius) that encompasses the geometric center of the complex and most of the area of the L935 dark nebula that is suspected to obscure the star. We have considered the stars whose apparent $K_{\mathrm{S}}$ magnitude and $\left(H-K_{\mathrm{S}}\right)$ color are consistent with them being
O-type stars up to a maximum distance of $1 \mathrm{kpc}$, and used an additional $J H K_{\mathrm{S}}$ color-color criterion that excludes most latetype, reddened stars. We have defined in this way a preliminary sample of 19 possible candidates for which low-resolution $H$ and $K$ band spectroscopy has been obtained. Most of these spectra correspond to cool photospheres, mainly belonging to AGB and carbon stars, as expected from the infrared photometric properties of those objects. However, three of our objects do display spectroscopic characteristics of early- or mid-type stars. Two of them are ruled out as possible ionizing stars, one being a foreground F5V star and the other a background early A-type giant as confirmed by its spectrum in the visible.

The third and brightest early-type object, J205551.3+435225, has both photometric and spectroscopic characteristics in the infrared consistent with it being a O5V star at the commonly accepted distance of the complex, $600 \mathrm{pc}$, obscured by $A_{V} \simeq 9.6$. We have been able to reliably confirm this spectral classification by means of a spectrum in the blue visible region, which excludes a spectral type O6 or later. The properties of a nearby ROSAT-detected source that we tentatively identify as the X-ray counterpart of J205551.3+435225 are also consistent with those of a typical O5V star. The location of this star, just East of the "Florida Peninsula" region of the North America nebula, is near the geometric center of the complex and very close to the accurately predicted position made from ridge structures observed in radio continuum. The fact that no other stars with spectral types B2V or earlier are found within $0^{\circ} 5$ of J205551.3+435225 indicates that this star does not highlight the existence of a cluster or a region of enhanced stellar density.

The spectral classification of J205551.3+435225 as a O5V star, its close proximity to the expected location, the good match between its 2MASS photometry and the characteristics expected in a $05 \mathrm{~V}$ star at the distance of the complex, and the fact that it is the only candidate fulfilling all these conditions despite the large area over which our search has been conducted, lead us to consider it very likely that the long history of the search for the ionizing star of two of the best known nebulae has been finally completed.

Acknowledgements. We are very pleased to thank the staff of the Calar Alto observatory, and especially Mr. Santos Pedraz, Mr. Alberto Aguirre, Ms. Ana Guijarro, and Mr. Jesús Aceituno, for providing us once again with excellent support during our observing run in Calar Alto. We also appreciate the constructive comments of the referee, Prof. Dr. H.J. Wendker, leading to significant improvements of this paper. AP acknowledges support from the OPTICON Network. This research has made use of the SIMBAD database operated at CDS, Strasbourg, France. It also makes use of data products from the Two Micron All Sky Survey, which is a joint project of the University of Massachusetts and the Infrared Processing and Analysis Center/California Institute of Technology, funded by the National Aeronautics and Space Administration and the National Science Foundation. We retrieved the data of the Canadian Galactic Plane Survey as Guest Users of the Canadian Astronomy Data Centre, which is operated by the Dominion Astrophysical Observatory for the National Research Council of Canada's Herzberg Institute of Astrophysics. 


\section{References}

Bally, J., \& Reipurth, B. 2003, AJ, 126, 893

Bally, J., \& Scoville, J. Z. 1980, ApJ, 239, 121

Berghöfer, T. W., Schmitt, J. H. M. M., \& Cassinelli, J. P. 1996, A\&AS, 118, 481

Berghöfer, T. W., Schmitt, J. H. M. M., Danner, R., \& Cassinelli, J. P. 1997, A\&A, 322, 167

Bessell, M. S., \& Brett, J. M. 1988, PASP, 100, 1134

Cambrésy, L., Beichman, C. A., Jarrett, T. H., \& Cutri, R. M. 2002, AJ, 123

Comerón, F., Pasquali, A., Rodighiero, G., et al. 2002, A\&A, 389, 874

Drilling, J. S., \& Landolt, A. U. 2000, in Allen's Astrophysical Quantities, ed. A. N. Cox (AIP Press)

Eiroa, C., Hefele, H., \& Zhong-yu, Q. 1983, A\&A, 54, 309

Fehrenbach, C. 1961, Publ. Obs. Haute-Provence, 5, 54

Goudis, C. 1976, Ap\&SS, 39, 173

Goudis, C., \& Meaburn, J. 1973, A\&A, 26, 65

Hanson, M. M. 2003, ApJ, 597, 957

Hanson, M. M., Conti, P. S., \& Rieke, M. J. 1996, ApJS, 107, 281

Herbig, G. H. 1958, ApJ, 128, 259

Hubble, E. P. 1922, ApJ, 56, 400

Jaschek, C., \& Jaschek, M. 1987, The classification of stars (Cambridge Univ. Press)
Jordi, C., Figueras, F., Torra, J., \& Asiain, R. 1996, A\&AS, 115, 401

Laugalys, V., \& Strayzis, V. 2002, Balt. Astron., 11, 205

Lynds, B. T. 1962, ApJS, 7, 1

Matthews, H. E., \& Goss, W. M. 1980, A\&A, 88, 267

Matthews, H. E., Baars, J. W. M., Wendker, H. J., \& Goss, W. M. 1977, A\&A, 55, 1

Meyer, M. R., Edwards, S., Hinkle, K. H., \& Strom, S. E. 1998, ApJ, 508, 397

Neckel, Th., Harris, A. W., \& Eiroa, C. 1980, A\&A, 92, L9

Oliva, E., \& Origlia, L. 1992, A\&A, 254, 466

Rieke, G. H., \& Rieke, M. J. 1985, ApJ, 288, 618

Schaerer, D., \& de Koter, A. 1997, A\&A, 322, 598

Sharpless, S., \& Osterbrock, D. 1952, ApJ, 115, 89

Taylor, A. R., Gibson, S. J., Peracaula, M., et al. 2003, AJ, 125, 3145

Tokunaga, A. T. 2000, in Allen's Astrophysical Quantities, ed A. N. Cox (AIP Press)

Voges, W., Aschenbach, B., Boller, Th., et al. 1999, A\&A, 349, 389

Walborn, N. R., \& Fitzpatrick, E. L. 1990, PASP, 102, 379

Wendker, H. 1968, Z. Astrophys., 68, 368

Wendker, H. J., Benz, D., \& Baars, J. W. 1983, A\&A, 124, 116

Westerhout, G. 1958, Bull. Astron. Inst. Netherlands, 14, 215 
F. Comerón and A. Pasquali: The ionizing star of the North America and Pelican nebulae, Online Material $p 1$

\section{Online Material}


F. Comerón and A. Pasquali: The ionizing star of the North America and Pelican nebulae, Online Material p 2

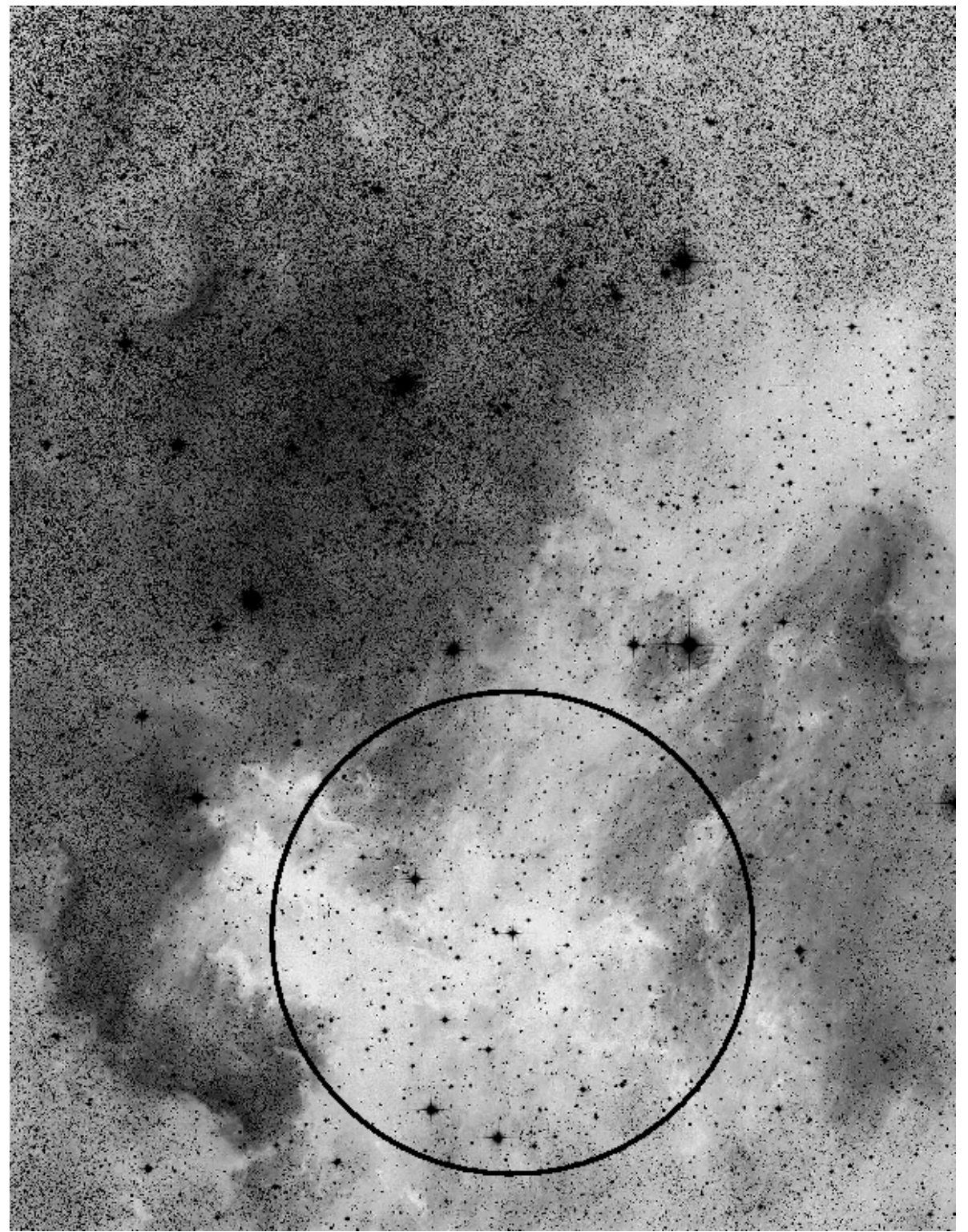

Fig. 1. The region of the North America (NGC 7000) and the Pelican (IC 5070) nebulae with the $0^{\circ} 5$-radius circular area where we have searched for the ionizing star, containing most of the L935 dark cloud. The image, courtesy of the German-Spanish Astronomical Center, is a negative print of an image obtained with the Schmidt telescope on Calar Alto. 